QUADERNS DE FILOSOFIA VOL. VIII NÚM. I (202 I): I I-35

eISSN: 234I-3042 DOI: IO.7203/QFIA. 8.I.I 5786

Mariela Destéfano

Universidad de Buenos Aires

Consejo Nacional de Investigaciones Cientificas y Tecnológicas

\title{
Las representaciones conceptuales en la ciencia cognitiva ${ }^{1}$
}

\author{
Conceptual Representations in Cognitive Science
}

Recibido: 13/9/2019. Aceptado: 22/4/2021

Resumen: En el marco de la ciencia cognitiva se ha polemizado acerca de la manera en la que el enfoque enraizado de la cognición pueda compatibilizarse con al enfoque clásico con el fin de explicar nuestras capacidades conceptuales. Sin embargo este debate metodológico no ha sido acompañado de una elucidación de la noción de "representación conceptual" tal como venía siendo entendida al menos en la filosofía y psicología cognitivas. El objetivo de este trabajo es analizar en que consiste una representación conceptual con el fin de aportar claridad al debate sobre las capacidades conceptuales enraizadas. Desarrollaré y evaluaré la idea de que una representación es conceptual si puede combinarse sistemáticamente para formar nuevas estructuras representacionales y si se puede utilizar en tareas psicológicas con independencia del estímulo.

Abstract: In cognitive science, it is an open debate whether grounded cognition might be compatible with traditional views of cognition. However, as far as I am aware, this methodological debate has not been accompanied by an elucidation of the notion of "conceptual representation" as it has been understood in philosophy and cognitive psychology. The aim of this paper is to offer an elucidation of this sort. I will develop and evaluate the idea that a representation is conceptual when it can be systematically combined to form new representational structures and when it can be used in psychological tasks regardless of the stimulus.

\footnotetext{
${ }^{1}$ Quisiera agradecer a los evaluadores de la revista por los fructíferos comentarios que me han permitido mejorar la presentación de este artículo.
} 
Palabras clave: capacidades conceptuales, sistematicidad, independencia del estímulo, cognición enraizada, psicología cognitiva.

Keywords: conceptual abilities, sistematicity, independence of stimulus, grounded cognition, cognitive psychology.

\section{INTRODUCCIÓN}

$\mathrm{E}$

N EL MARCO DE LA CIENCIA COGNITIVA se ha propuesto que nuestras capacidades conceptuales estarían enraizadas en otras capacidades tales como las sensitivas, perceptivas, motoras y afectivas (BArsalou 20 i6; Pecher 20 i 3; Martin 20i6; Goldinger 20i6; Mahon 20i5). Según Weber y Vosgerau (2018), "enraizado" puede interpretarse tanto en términos de condiciones de adquisición como en términos de condiciones de constitución. En el primer caso, una habilidad cognitiva A está enraizada en la base de B si B es necesario para adquirir A. En el segundo caso, una habilidad cognitiva A está enraizada en B si B es necesario para poseer la habilidad A. Muchos desarrollos en el enfoque enraizado se focalizan en el rol de las simulaciones en la cognición, entendiendo por simulación la reactivación de los estados perceptivos, motores e introspectivos adquiridos durante la experiencia (BARSALOU 2008). En el transcurso de las experiencias (e.g. usar una silla) el cerebro captura información a través de las distintas modalidades perceptivas y la integra en una representación multimodal almacenada en la memoria. Luego, cuando en aquellas tareas que demanden representar un concepto (e.g. SILLA), estas representaciones multimodales capturadas durante la experiencia son reactivadas.

Más allá de las particularidades que tengan las distintas propuestas enraizadas, todas se contraponen al enfoque clásico según el cual a las capacidades conceptuales subyacen representaciones que son independientes de los sistemas modales del cerebro para la percepción, la acción, afectividad e introspección (BARSAlOu 20Io). Sin embargo, es improbable que el enfoque enraizado tenga éxito sin el compromiso con elementos propios del enfoque clásico según el cual las representaciones conceptuales son simbólicas (BARSALOU 2008). De este modo, uno de los grandes objetivos explicativos de los defensores de la cognición enraizada es encontrar el modo de compatibilizar ambos enfoques. En este sentido, en los últimos años ha tenido lugar un debate metodológico (que denominaré "debate enraizado) el cual es prolífico y ha generado una pluralidad de posiciones respecto de cómo entender la integración entre en enfoque enraizado y el clásico.

Por un lado, una de las maneras de entender dicha integración es considerar que las representaciones simbólicas y las simulaciones operan en una 
misma arquitectura cognitiva. Este es el caso de propuestas teóricas como las de Dove (2009), Louwerse (2012) o Zwan (2014) para quienes los procesos conceptuales involucran ambos tipos de representaciones. Por ejemplo, Zwan ha propuesto que las representaciones simbólicas se utilizan en tareas que demandan mayor nivel de abstracción mientras que las simulaciones se utilizarían en las interacciones concretas con el ambiente. De acuerdo con esta manera de entender la integración ambos tipos de representaciones pertenecen al mismo nivel funcional. Frente a ello, autores como Barsalou (2010) entienden que las simulaciones podrían constituir una propuesta de implementación neural de una arquitectura simbólica clásica. Aunque su teoría pertenece originariamente a la psicología cognitiva, Barsalou entiende que el enfoque enraizado se acomoda "naturalmente" a la investigación cerebral "debido a que toma en consideración las modalidades perceptivas" (2008, 635). En este caso, ambos enfoques se integrarían pero desde diferentes niveles explicativos, debido a que el enfoque enraizado constituiría una implementación cerebral de las propiedades funcionales que tendría una arquitectura clásica de representaciones simbólicas.

Hasta lo que sé, este debate metodológico no ha sido acompañado de una elucidación de la noción de "representación conceptual" tal como venía siendo entendida al menos en la filosofía y psicología cognitivas. Por esta razón, el objetivo de este trabajo es analizar en que consiste una representación conceptual con el fin de aportar claridad conceptual al debate sobre las capacidades conceptuales enraizadas. Hacer jugar en este debate un criterio de mínima de representación conceptual sustentada por la literatura sobre el tema contribuiría, en parte, a clarificar algunos elementos de juicio a favor de una u otra posición en el debate sobre cognición enraizada. Pues, habiendo elucidado cuáles son las condiciones para una representación conceptual, el hecho de que tanto representaciones simbólicas como simulaciones cumplan con dichas condiciones podría significar que ambos tipos de representaciones operarían conjuntamente en una misma arquitectura cognitiva aunque haciendo diferentes aportes en tareas cognitivas diferentes. Por otro lado, un análisis conceptual de las simulaciones que muestre que no cumplen con ciertas condiciones mínimas de conceptualidad podría indicar o bien que pertenecen a un nivel de implementación neuronal o bien que hacen un aporte a las arquitectura cognitivas simbólicas pero en carácter de representaciones no conceptuales. De hecho, más adelante abriré la posibilidad de considerar a las simulaciones o bien como representaciones no conceptuales o bien como entidades neuronales. Es cierto que profundizar en estos enfoques es algo que excede este trabajo y que demanda evidencia empírica. Lo importante en este contexto es que ambas interpretaciones tendrían lugar de momento que las simulaciones sólo cumplirían parcialmente con los criterios de conceptualidad presentados. 
Existe un consenso filosófico en sostener que los contenidos de los estados de actitud proposicional (por ejemplo, lo creído en el caso de las creencias o lo deseado en el caso de los deseos) están constituidos por conceptos, en la medida en que estar en un estado de actitud proposicional requiere la posesión de los mismos. Por ejemplo, un individuo no puede creer (ni formular el juicio de) que el gato es perezoso sin poseer, al menos, los conceptos GATO y PEREZOSO. Es cierto que la literatura sobre conceptos involucra una diversidad de discusiones planteadas tanto en el plano filosófico como psicológico. En la filosofía ha sido usual considerar a los conceptos como entidades abstractas que carecen de especificaciones psicológicas (FrEge I892). Frente a ello están los debates psicológicos sobre conceptos que en su mayoría han asumido una ontología de particulares mentales. Como el objetivo de este trabajo es focalizarse en los desarrollos de las ciencias cognitivas donde suele adherirse a una perspectiva de particulares mentales en este trabajo se adoptará la idea de que los conceptos son representaciones mentales, dejando de lado planteos que desnudan a los conceptos de todo factor psicológico. En este marco el contenido de los estados de deseos y creencias está constituido por conceptos, entendidos como representaciones que son particulares mentales instanciados físicamente, que poseen propiedades tales como activarse y procesarse de manera independiente del estímulo y combinarse satisfaciendo el requisito de generalidad (Evans I982). ${ }^{2}$ En este trabajo desarrollaré y evaluaré la idea de que una representación es conceptual si puede combinarse sistemáticamente según el requisito de generalidad para formar nuevas estructuras representacionales y si se puede utilizar en tareas psicológicas (e.g. categorización, razonamiento práctico, etc.) con independencia del estímulo. En la sección 2 haré explicito que rescato una caracterización funcional de las representaciones conceptuales. En la sección 3 presentaré la idea de que los conceptos son representaciones independientes del estímulo y diré algunas palabras relativas a la posibilidad de que la noción de "simulación" respete este criterio de conceptualidad. En la sección 4 desarrollaré la idea de que son representaciones que se combinarían sistemáticamente y también evaluaré en qué sentido las simulaciones no se combinarían de esta manera. En ambas secciones focalizo en la noción de "simulación perceptiva" propia de Barsalou (I999A; I999B) debido a la centralidad que tiene dicha noción en el enfoque enraizado. Si bien es un concepto

${ }^{2}$ Cabe aclarar que existe una significativa interacción entre los enfoques filosóficos y psicológicos acerca de los conceptos (LaURence y Marglis I 999). Lingüistas y psicólogos se han inspirado en los aportes wittgenstanianos sobre los parecidos de familia o en la distinción fregeana entre sentido y referencia, o en las tesis extenalista y esencialistas de Kripke y Putnam para el planteo de su teorías empíricas. También muchos debates filosóficos se han enriquecido con los aportes de la psicología acerca de las tareas de categorización. Esta retroalimentación ha permitido incorporar aportes de filósofos como Evans en el marco de las ciencias cognitivas. 
que pertenece a la psicología cognitiva es compatible con otras propuestas enraizadas de la ciencia cognitiva como por ejemplo la de Prinz (2002). En las conclusiones retomaré los lineamientos generales presentados en las anteriores secciones e indagaré brevemente sobre las razones por las cuales el debate enraizado se vería enriquecido con este análisis de las condiciones de mínima para las representaciones conceptuales.

\section{El ROL FUNCIONAL DE LOS CONCEPTOS}

Generalmente, el criterio para determinar qué tipo de entidades son los conceptos apela al rol funcional que este tipo de representaciones debería satisfacer en nuestra vida mental. Hacer una caracterización funcional de una entidad significa caracterizarla en términos de sus propiedades funcionales. Las propiedades funcionales son las que describen lo que hace una entidad, cuál es su rol en un sistema más amplio (Weisкopf ms.). Así, caracterizar funcionalmente a los conceptos significa dar una descripción de cuáles son los roles que debe satisfacer una entidad para ser concepto: "algo es un concepto en virtud de la función que desempeña en un sistema cognitivo" (Lalumera 20Io, 217). Una vez que se especifican estos roles, todas aquellas entidades que los satisfacen serían conceptos. Los roles funcionales que deberían satisfacer los conceptos varían según las particularidades de cada propuesta teórica. Esta especificación funcional de las representaciones conceptuales pretende rescatar lo que, en un sentido general, filósofos y psicólogos han entendido por "concepto".

Por ejemplo, Prinz considera que los conceptos tienen que cumplir el rol de constituirse como representaciones que están bajo control endógeno: "Sugiero que los conceptos son espontáneos: son representaciones que pueden estar bajo control endógeno del organismo" (2002, 197). Una representación bajo control endógeno es activada y manipulada por el sujeto que la posee. En este sentido, una representación bajo control endógeno es consciente para el sujeto que la posee, teniendo en cuenta que para Prinz (2004) la posibilidad de control del organismo involucra un acceso consciente a la representación. Asimismo, una representación bajo control endógeno se produce voluntariamente, en tanto que "voluntariamente se pueden formar pensamientos usando conceptos" (Prinz 2004, 46). Pero decir que los conceptos son representaciones manipuladas de manera consciente y voluntaria, dificultaría la posibilidad de afirmar que hay sistemas de procesamiento temprano de la información (como el lingüístico) que operan sobre conceptos. Estos sistemas de procesamiento operarían sobre representaciones subpersonales las cuales no pueden 
ser manipuladas consciente y voluntariamente por un sujeto. Por lo tanto, la razón para dejar de lado la caracterización de Prinz es que no constituye una condición de mínima que pueda aplicarse a la amplitud de casos de conceptualidad (que va del nivel personal al subpersonal) que existe en la psicología y la filosofía de la ciencia cognitiva.

Weiskopf (ms.), por su parte, asigna a los conceptos un rol funcional diferente:

La función de los conceptos es permitir a una criatura que los tiene trascender las maneras de representar el mundo que sus otros sistemas cognitivos le proporcionan, especialmente, los sistemas sensorio-motores (13)

Entendidos de esta manera, los conceptos son representaciones abstractas que representan propiedades que no podrían ser representadas por los sistemas sensorio-motores:

Hay muchas cosas que no pueden ser representadas por nuestros sistemas dedicados para la percepción, [...] y el lenguaje, etc. Los espacios de Hilbert, los objetos matemáticos, no tienen manifestaciones perceptivas y, seguramente, exceden los poderes de nuestra facultad numérica. La propiedad de ser una ilusión del Infierno de Dante es algo que la facultad del lenguaje no puede representar (WeISKopf ms., 12)

Así, los conceptos también serían representaciones abstractas en el sentido de que pueden representar las mismas propiedades representadas por los sistemas sensorio-motores pero de manera diferente. Por ejemplo, puede haber conceptos de cualidades perceptivas que no las representan "bajo un modo de presentación perceptivo" (Weiskopf ms., 13). Considero que el criterio de abstracción es correcto, sin embargo, según mi entender, no parece ser un criterio de mínima que hace que una representación sea conceptual. Más adelante adoptaré como una de las condiciones de mínima para la conceptualidad la independencia del estímulo. Podría especularse que el carácter abstracto de las representaciones conceptuales estaría posibilitado por su independencia del estímulo. Aunque no me detendré sobre esta cuestión, puede pensarse que una representación es abstracta en tanto que, en algún sentido, puede instanciarse sin que sea activada por un estímulo perceptivo. De este modo, la abstracción no sería un criterio de mínima para la conceptualidad sino que dependería, en parte, del de la independencia del estímulo. Además, considerar a la abstracción como una condición de mínima para la conceptualidad significaría alejarse de la literatura psicológica y filosófica de raigambre enraizada. 
Otra manera de entender el rol funcional de los conceptos es a partir de la siguiente caracterización que hace Machery:

Un concepto de $x$ es un cuerpo de conocimientos sobre $x$ que está almacenado en la memoria de largo plazo y se usa por default en los procesos subyacentes a la mayoría de las competencias cognitivas superiores, cuando estos procesos resultan en juicios sobre $x(2009,12)$

Según esta caracterización, los conceptos son representaciones almacenadas en la memoria de largo plazo. Sin embargo, muchos filósofos y psicólogos defienden la posibilidad de que los procesos subyacentes a las competencias cognitivas superiores, de las que habla Machery, operen sobre representaciones temporalmente construidas en la memoria de trabajo (BARSALOU I999A; Prinz 2002; Weiskopf ms.). Tal como sucede con la propuesta de Weiskopf, asumir este criterio como condición mínima de conceptualidad significaría alejarse de muchas de las propuestas enraizadas que serán relevantes en este trabajo.

Desarrollaré dos criterios funcionales de mínima para la conceptualidad que se distancian de los propuestos por los autores anteriores. Según estos criterios, ciertas representaciones son conceptuales porque se emplean en una variedad de tareas cognitivas donde suele atribuirse conceptos con independencia del estímulo y se pueden combinar sistemáticamente para formar nuevas estructuras. La primera condición es la que han propuesto sobre todo los psicólogos y la segunda condición es la que han propuesto los filósofos. El motivo que llevaría a establecer condiciones de conceptualidad que han impuesto tanto psicólogos como filósofos es que esta noción de "concepto" sea útil desde un punto de vista filosófico y empírico. Es decir que estas condiciones no son arbitrarias sino que, por el contrario, suelen aparecer en las propuestas que caracterizan al contenido conceptual. De esta manera, esta noción de "concepto" es descriptiva porque rescata lo que la mayoría de los psicólogos y filósofos entienden por "concepto", aunque no haya mucho acuerdo entre ellos ni al interior de cada disciplina.

En psicología cognitiva, no se utiliza un vocabulario unificado para referirse a conceptos. Algunos usan términos que provienen de la inteligencia artificial, como "frames" o "scripts", otros usan "conceptos" y "categorías" de manera intercambiable (SMith y Medin I98I). Y las diferencias en el tratamiento de los conceptos van más allá de lo meramente terminológico. Existen muchas teorías psicológicas que se diferencian en el tipo de información que consideran que constituye la estructura del contenido de un concepto. Según algunas de estas teorías, los conceptos pueden ser prototipos (RosH I978), 
ejemplares (Estes 1994) o teorías (Rehder 2003). Pero a pesar de estas diferencias, todas las investigaciones psicológicas sobre representaciones conceptuales acuerdan en sostener que la información conceptual es la que interviene en tareas como categorización, comprensión y producción lingüística (PICCINinI y SCOTT 2006).

La condición filosófica acerca de la conceptualidad, según la cual los conceptos pueden recombinarse de manera sistemática para formar otras estructuras, es lo que rescata Evans (1982) con el requisito de generalidad. El requisito de generalidad surge al prestar atención a la estructura de los pensamientos. Los pensamientos tienen una estructura que refleja las relaciones entre los constituyentes de las oraciones del lenguaje.

\section{Conceptualidad e independencia del estímulo}

Para caracterizar a los conceptos "tenemos que atender al rol que tienen en las teorías psicológicas contemporáneas" (PiCcinini y ScOTt 2006, 295). No es controversial sostener que el contenido conceptual es de vital importancia en tareas cognitivas que Piccinini y Scott (2006) identifican en la siguiente lista:

- Discriminación: responder de manera diferenciada a objetos, propiedades y eventos que tienen algo saliente en común.

- Categorización: asociar de manera arbitraria un input con una respuesta, incluyendo la habilidad de producir una etiqueta apropiada (e.g., una palabra) en respuesta a una clase de inputs, así como también elegir el objeto o comportamiento apropiado en respuesta a una etiqueta dada como estímulo (Rosh 1999).

- Inferencias no lingüísticas: establecer inferencias sobre clases de objetos en el mundo. Puede incluir el razonamiento deductivo (Braine et al., 1984), analógico, (GentNer 1983), razonamientos prácticos (GREene 2009), entre otros.

- Inferencias lingüísticas: establecer inferencias que incluyen palabras (e.g., la inferencia que va de "rojo" a "coloreado") e inferencias entre oraciones en virtud de su gramática.

- Comprensión y producción lingüística: procesar las palabras y las oraciones ante un input lingüístico de habla o escritura (Ellis y Young 1992). ${ }^{3}$

${ }^{3}$ Los componentes no conceptuales también intervienen en algunas de las tareas que aparecen en esta lista. Ver nota 7 en Piccinini y Schott (2006). 
Esta caracterización sigue de cerca la noción de "concepto" que puede extraerse de la psicología cognitiva. Aunque es difícil identificar una noción unívoca de concepto compartida por las diferentes teorías psicológicas (MACHERY 2009), en un sentido general, todas las teorías de conceptos estarían dispuestas a sostener que estos son cuerpos de información usados en tareas como categorización, deducción, inducción, etc. Los psicólogos suelen entender que, por ejemplo, las tareas en las que el sujeto tiene que realizar inferencias no lingüísticas y lingüísticas requieren la participación de conceptos. Tomemos la siguiente inferencia: "Si se da $X$ e $Y$, entonces no se da $Z$. Por lo tanto, si se da $Z$, o no se da $X$ o no se da $Y$ " (Monti et al. 2009). Es una inferencia deductiva en la que se llega a la conclusión haciendo uso de reglas lógicas y utilizando conceptos como $X, Y$ y $Z$. Tomemos ahora la siguiente inferencia lingüística: "Era $X$ lo que $Y$ vio tomar a $Z$. Por lo tanto, $Z$ fue visto por $Y$ tomando $X$ " (Monti et al. 2009). En este caso, la inferencia es lingüística porque se realiza en virtud de la gramática de las oraciones y no en virtud de reglas lógicas. También en esta oportunidad la inferencia se establece utilizando conceptos como $X, Y$ y $Z$.

Las representaciones que subyacen a las tareas cognitivas como las anteriores son conceptuales en el sentido de que satisfacen lo que Millikan (2000) denominó "requisito de distancia". Desde esta perspectiva, una representación es conceptual en tanto que puede activarse y procesarse con independencia del estímulo. Esto quiere decir que aquello en el mundo que activaría el concepto no es necesario para su ocurrencia, lo cual plantea una separación entre la representación y el input de tal manera que puede darse la ocurrencia de la primera sin la ocurrencia del segundo (CAMP 2009). Este criterio para la conceptualidad concierne a la relación que tiene una representación con aquello que la instancia: una representación sería conceptual cuando puede activarse y procesarse con independencia de los estímulos del mundo. Por supuesto que hay circunstancias en las que, por ejemplo, PERRO se activa y procesa en caso de que haya algún perro como estímulo distal. Pero si, en ciertas tareas cognitivas, PERRO puede activarse y procesarse sin la necesidad de que haya un perro como estímulo, entonces podría pensarse que esta representación es conceptual.

Una representación es independiente del estímulo o bien porque el estímulo no está presente, o bien porque el estímulo está presente pero podría no intervenir en la activación y el procesamiento de la representación. En este sentido, la independencia del estímulo se manifiesta de dos maneras, tanto en circunstancias en las que efectivamente no está el estímulo pertinente como en circunstancias en las que puede estar el estímulo pertinente. Quisiera explorar con algún detalle ambas formas de la independencia del estímulo. En primer lugar, el estímulo de la representación no está presente en el sentido de no encontrarse en el ambiente circundante al organismo que posee el concepto. Las 
representaciones cuyos estímulos se encuentran en ambientes lejanos se usan en cierta clase de razonamiento espacial. Imaginemos el siguiente razonamiento espacial. El objeto $A$ está a la distancia $X$ de donde estoy. El objeto $B$ está cerca del objeto $A$, por lo tanto el objeto $B$ está a la distancia $X$ de donde estoy. Este razonamiento exige representaciones del objeto $A$ y el objeto $B$, cuyos estímulos no se encuentran en el ambiente inmediato dado que están a la distancia $X$.

A su vez, el estímulo de una representación puede no estar presente en el sentido de que dicho estímulo no existe. Los razonamientos prácticos de toma de decisión exigen la utilización de representaciones independientes del estímulo en este sentido. En un razonamiento de toma de decisión, un individuo elige uno entre dos o más posibles cursos de acción. Decidir no es simplemente seleccionar uno de estos cursos de acción: "una genuina toma de decisión involucra la selección entre diferentes cursos de acción basada en las consecuencias probables que esos cursos de acción tendrían" (BERMúdez 2003, 124). Es decir que una toma de decisión involucra algún tipo de proceso de comparación entre diferentes cursos de acción y toma en cuenta sus posibles consecuencias. Tal como sostiene Bermúdez: "esto requiere representar los diferentes resultados potenciales, representar la utilidad que tiene cada uno de ellos, y estimar la probabilidad de que cada resultado ocurra" $(2003,125)$. Lo importante a destacar es que la representación de los diferentes resultados potenciales o la representación de la utilidad que tienen las consecuencias de los cursos de acción que se evalúan en la toma de decisión se activan aun cuando en el mundo no haya un estímulo distal que produzca su instanciación.

En segundo lugar, una representación también es independiente cuando el estímulo puede estar presente en el ambiente circundante al organismo que posee el concepto y aun así, no activa la representación en cuestión. Este tipo de representaciones independientes del estímulo son las necesarias para tener lo que denomino "pensamientos solipcistas". Es concebible la posibilidad de que un sujeto piense que los collies son bellos perros cuando en su ambiente circundante hay un collie, y sostener al mismo tiempo que este collie no ha sido necesario para la activación y el procesamiento de COLLIE por parte del sujeto. Este concepto ha sido autogenerado en el sentido de que el estímulo, aun cuando estaba presente, no lo ha provocado.

A diferencia de las representaciones independientes del estímulo, las representaciones dependientes del estímulo necesitan la presencia del estímulo pertinente para su activación y procesamiento. Son representaciones en las que hay una separación débil entre la representación y el estímulo dado que no puede darse la primera sin que el segundo sea el caso. Un ejemplo en el cual sería necesario postular representaciones dependientes del estímulo es cuando, en el reino animal, los animales machos evalúan a otros macho en una situa- 
ción de competencia (BERMÚDeZ 2003). Mediante la sola inspección del macho contrincante "el animal podría ver que pelear es una respuesta apropiada o inapropiada" (BERMúdez 2003, 121). Sus posibilidades de acción resultan de la sola inspección de las condiciones percibidas en el ambiente. El ambiente provee toda la información necesaria (e.g., un macho muy pequeño) para explicar la respuesta conductual de un animal (e.g., iniciar una pelea). Si se hace la concesión de que este tipo de conductas debe explicarse atribuyendo representaciones a los animales, estas representaciones deberían entenderse como dependientes del estímulo.

Otro ejemplo en el cual sería necesario atribuir representaciones dependientes del estímulo sería en el caso de los pacientes con síndrome de dependencia del estímulo. Este síndrome es producto de lesiones en el lóbulo frontal y se caracteriza por el hecho de que los pacientes se desempeñan en sus tareas cognitivas atendiendo de manera exclusiva a los estímulos ambientales inmediatos (Lhermitte i 986). Un ejemplo de ello es el paciente A.D. quien asumía diferentes roles según el contexto en el que estaba (Conchiglia et al. 2007). En sus discusiones con los psicólogos, A.D. sostenía ser un psicólogo y actuaba como tal. En el hospital, frente a los médicos, A.D. decía ser médico así como también, en ocasión de una visita a un edificio en construcción, A.D. sostenía ser ingeniero y asumía dicho rol. Esta singular conducta podría explicarse atribuyendo a A.D. representaciones que dependen del estímulo. Este paciente se representa ser un psicólogo, médico o ingeniero atendiendo de manera exclusiva a la información que le brinda el ambiente en el que está.

Es importante destacar que la distinción entre dependencia e independencia del estímulo es independiente respecto de la determinación internalista o externalista del contenido. Son concebibles todas estas opciones:

(i) Una representación dependiente del estímulo cuyo contenido está determinado de manera externalista.

(ii) Una representación dependiente del estímulo cuyo contenido está determinado de manera internalista.

(iii) Una representación independiente del estímulo cuyo contenido está determinado de manera externalista.

(iv) Una representación independiente del estímulo cuyo contenido está determinado de manera internalista.

La pregunta previa que surge es ¿por qué podemos admitir las opciones que van de (i) a (iv)? Porque una cosa es tomar partido respecto de cómo se activa y procesa una representación, y esto se relaciona con la presencia o ausencia del estímulo pertinente para la activación y procesamiento de la 
representación, y otra cosa es tomar posición respecto de la manera en que se individúa el contenido de la representación, y esto se relaciona estrictamente con la semántica de la representación. Quiero exponer esta idea tomando en consideración, sobre todo, las opciones (ii) y (iii).

La opción (ii) asume la posibilidad de una representación dependiente del estímulo cuyo contenido está determinado de manera internalista. Por ejemplo, una representación táctil que se ha activado a causa de la presencia de un objeto rugoso es dependiente del estímulo porque hubo un objeto en el mundo que dio lugar al procesamiento de dicha representación. Pero establecer esta relación entre el estímulo de la representación y la representación no obliga desde un punto de vista conceptual a adoptar una semántica externalista. El contenido de esta representación no necesariamente está determinado por el objeto rugoso en el mundo que causó la representación, también puede concebirse que este contenido esté determinado por el rol que tiene en relación a otras representaciones del sistema cognitivo, por ejemplo.

La opción (iii) es la que suele adoptar la mayoría de los filósofos y asume la posibilidad de una representación independiente del estímulo cuyo contenido está determinado de manera externalista. Por ejemplo, la representación conceptual PERRO sería independiente del estímulo en la medida en que puede darse la activación del concepto sin que haya ningún estímulo pertinente en el mundo que lo active. Sin embargo, aunque la representación no depende del estímulo para activarse, aun así se puede sostener que el contenido de PERRO está determinado por la propiedad de la perritud en el mundo.

Luego de haber presentado la primera condición de mínima para una representación conceptual la pregunta que surge es si las "simulaciones perceptivas", las cuales propone Barsalou (I999A; I999B) en el enfoque enraizado, son independientes del estímulo. Barsalou (I999B) define a las simulaciones como activaciones top-down de áreas sensorio-motoras que reactiva experiencias perceptivas. Éstas son creadas por mecanismos específicos denominados "simuladores" los cuales permiten al sistema cognitivo construir representaciones de "una entidad o eventos en su ausencia" (Barsalou i999, 586). Por ejemplo, en la construcción de la simulación de un auto, la atención selectiva se focaliza en diferentes aspectos del objeto los cuales quedan organizados en un registro perceptivo espacialmente organizado. La información perceptiva integrada en este registro servirá posteriormente para simular nuevas experiencias coherentes del objeto lo cual permitirá al sistema cognitivo anticiparse a nuevos encuentros con autos. El objeto simulado tiene aspectos multimodales de la experiencia que quedan incorporados en la múltiple información perceptiva (e.g. visual, táctil, auditiva) de las simulaciones. Dada esta caracterización de las simulaciones puede pensarse que en términos de cómo se construyen las mismas no 
serían independientes del estímulo. Pues, el ambiente contribuye causalmente en la genética de las simulaciones. Los diferentes aspectos de la experiencia reclutados por la atención selectiva tienen un rol central en la constitución de las mismas. En este sentido, las simulaciones carecerían de cualquier versión de independencia del estímulo de momento que son representaciones enraizadas en tanto que su adquisición involucra información perceptiva extraída del ambiente (Weber y Vosgerau 2O I 8).

Sin embargo existe un sentido interesante en el cual las simulaciones serían independientes del estímulo. Barsalou (2008) explicita que las simulaciones de hecho proceden independientemente de las experiencias sensoriomotoras concretas. La idea central de enfoque enraizado es que los mismos procesos perceptivos, afectivos y motores involucrados en la interacción con objetos y eventos, se activan cuando razonamos, comprendemos y pensamos en general acerca de esos objetos y eventos. Las tareas conceptuales (e.g. tareas de verificación de propiedades, razonamiento espacial, razonamiento abstracto, comprensión de palabras, entre otras) involucrarían simulaciones almacenadas en la memoria de trabajo (BARSALOU 2008). La particularidad que tienen las tareas conceptuales es que una vez superado el análisis perceptivo inicial los mecanismos que usan las simulaciones operan de manera independiente a cualquier estímulo. Por ejemplo, en una tarea de verificación de propiedades, donde los sujetos tienen que decidir si una propiedad (e.g. CUERNOS) pertenece a una categoría (CABALLOS), la utilización de simulaciones de los conceptos y sus propiedades que permiten desarrollar la tarea son procesadas con independencia de la ocurrencia real de objetos como cuernos o caballos. De modo que las simulaciones serían representaciones independientes del estímulo que se activan por el solo funcionamiento de los procesos conceptuales.

\section{Conceptualidad y ReQuisito DE GENERAlidad}

La segunda caracterización de las representaciones conceptuales es un requisito filosófico que surge de la literatura analítica. El contenido conceptual es aquel cuyos constituyentes se pueden combinar sistemáticamente para formar nuevas estructuras representacionales. Esta idea fue presentada por Evans $(\mathrm{I} 982,104)$ en lo que denomina el "requisito de generalidad":

Si a un sujeto se le atribuye el pensamiento de que $a$ es $F$, entonces debe tener los recursos conceptuales para tener el pensamiento de que $a$ es $G$, para cualquier propiedad de ser $G$ de la cual tiene una concepción. Esta es la condición que llamo "El requisito de generalidad" 
Los filósofos suelen sostener que cierto tipo de pensamientos están estructurados. Los pensamientos Helena es salvaje y Arístides es mimoso. El contenido de estos pensamientos está constituido por conceptos como HELENA y SALVAJE, en el primer caso, y ARÍSTIDES y MIMOSO, en el segundo caso. Estos pensamientos están estructurados en tanto que su contenido, constituido por conceptos, responde a las formas $F a$ y $G b$, en donde $a$ es HELENA, $F$ es SALVAJE, $b$ es ARÍSTIDES, y $G$ es MIMOSO. Lo que plantea Evans (I982) con el requisito de generalidad es que si un sujeto tiene los pensamientos estructurados $F a$ y $G b$, entonces no habría ningún impedimento conceptual para que tenga los pensamientos $G a$ y $F b$. La idea es que la posibilidad de tener nuevos pensamientos está dada por la recombinación de los constituyentes de otros pensamientos del sujeto. Así, el pensamiento $F a$ sería la intersección entre dos series, la de $F a, F b, \ldots$, y la de $F a, G a, \ldots$ Asimismo, el pensamiento $G b$, sería la intersección entre dos series, la de $G a, G b, \ldots$, y la de $F b, G b \ldots$ (Evans I982, 104).

Evans extrae la idea de que tener ciertos pensamientos nos compromete con la posibilidad de tener otros pensamientos a partir de lo que sucede en el caso de las oraciones del lenguaje natural. En este caso, si un sujeto comprende " $F a$ " y " $G b$ ", entonces debemos comprometernos con la idea de que el sujeto también comprenderá " $F b$ ” “ " $G a$ ". Evans establece una analogía según la cual, si las oraciones del lenguaje natural y los pensamientos comparten la característica de estar estructurados, y si la comprensión de ciertas oraciones involucra la comprensión de otras oraciones, entonces tener ciertos pensamientos involucra tener otros pensamientos. Los límites que Evans ve en esta analogía es que mientras que los pensamientos están esencialmente estructurados, las oraciones del lenguaje natural no estarían esencialmente estructuradas. ${ }^{4}$

Pero Evans $(1982,101)$ va más allá y sostiene que la capacidad de combinar los constituyentes de los pensamientos es lo que constituye tener un concepto: "Así, alguien que piensa que Juan es feliz y que Harry es feliz ejercita en dos ocasiones la habilidad conceptual que llamamos poseer el concepto de "felicidad". Evans analiza la posesión de conceptos en términos de las habilidades conceptuales que ejerce el sujeto. De manera que alguien que piensa que Juan es feliz y que Harry es feliz ejercita en dos ocasiones la misma "habilidad conceptual" que consiste en poseer el concepto FELIZ. Poseer un concepto consiste en esta capacidad (o habilidad conceptual, en términos de Evans) de combinarlo de manera irrestricta con otros conceptos. Cuando decimos que

${ }^{4}$ Esto es así en el caso de las oraciones del lenguaje natural porque, según Evans (1982, 102), "un significado expresado por una oración estructurada también puede expresarse con una oración no estructurada". Por ejemplo, una oración de una palabra introducida por estipulación para tener tal y cual significado. 
un sujeto posee el concepto FELIZ, decimos que no hay ninguna restricción para que el sujeto piense Juan es feliz, Harry es feliz, Susana es feliz, Maria es feliz, etc. (teniendo en cuenta que posee los conceptos JUAN, HARRY, etc.).

Siguiendo a Ryle (1949), las habilidades, sean conceptuales o no, son propiedades disposicionales. Una propiedad disposicional no se encuentra en un estado particular, sino que es susceptible de encontrarse en un estado particular cuando se da determinada condición. Por ejemplo, que algo sea frágil no consiste en que está actualmente roto, sino que consiste en que, si fuese sometido a golpes o forcejeos, se rompería. En el caso específico del desempeño en ciertas habilidades conceptuales, aceptándose que las habilidades son disposiciones, no es un asunto de lo que efectivamente se hace, sino de lo que se es capaz de hacer con ese concepto (Fodor I998, 19).

Las disposiciones son propiedades que pueden actualizarse bajo determinadas condiciones. En general, para cada disposición hay actualizaciones que parecen ser típicas. Así, la manera típica en que se actualiza la fragilidad de un objeto es rompiéndose cuando se lo somete a golpes. Pero quizá haya maneras menos típicas de actualización de la disposición de ser frágil. Someter un objeto a determinadas condiciones de calor que traigan como consecuencia una alteración en su estructura, tal vez sea una manera de actualizar su fragilidad. Lo que me importa resaltar es que las disposiciones pueden actualizarse de diferentes maneras gracias a su base categorial. La base categorial de una propiedad disposicional está constituida por las propiedades microestructurales que son causalmente responsables, en ciertas circunstancias, de las distintas manifestaciones de la disposición (FARA 2006).

Las habilidades conceptuales, de las que habla Evans, en tanto disposiciones tendrían una base categorial. La base categorial de las habilidades conceptuales es la que tiene la eficacia causal para producir las diferentes manifestaciones de estas habilidades. Evans (1982) admite que hay "algo en común" entre el pensamiento $F a$ y $G a$, en el sentido de que cada vez que se ejercita $a$ debe haber una causa común para esa habilidad. Con todo, Evans no hace un tratamiento de las habilidades conceptuales poniendo peso en su base categorial. Para poder decir algo más en relación a la base causal que subyace a las habilidades conceptuales habría que alejarse de la presentación que hace Evans del requisito de generalidad.

Tenemos que explicar de dónde surgen los poderes causales comunes que permiten pensar $F a, F b, G a$ y $G b$. Se puede pensar que las habilidades de pensar $F a, F b, G a$ y $G b$ se dan gracias a las representaciones conceptuales que tienen el poder causal de generar estas distintas combinaciones. Estas representaciones tienen ese poder causal debido a sus propiedades sintácticas. Y las propiedades sintácticas/causales son propiedades del vehículo de las representaciones. Según esta 
lectura, el requisito de generalidad es un requisito que satisfacen los vehículos de los pensamientos (BeCK 20I2). Esta es la lectura que adopta Fodor y Phylyshyn (1988) del requisito. A diferencia de Evans, para quien poseer un pensamiento consiste en ejercer ciertas habilidades cognitivas estructuradas, para Fodor poseer un pensamiento consiste en la combinación de particulares mentales.

La caracterización filosófica de los conceptos a partir del requisito de generalidad es insuficiente si tenemos en cuenta la investigación empírica. Difícilmente puedan encontrarse referencias al requisito de generalidad en los estudios psicológicos sobre categorización y otras tareas cognitivas en las que intervienen conceptos. Como creo que una buena manera de abordar las representaciones conceptuales es atendiendo tanto a una especificación filosófica como psicológica en lo que sigue desarrollaré en qué sentido el requisito de sistematicidad se asocia a un aspecto fáctico de nuestra vida cognitiva. Será la propuesta de Jerry Fodor la que nos ayude a tal propósito. El requisito de generalidad expresa, en parte, la propiedad de la sistematicidad que Fodor (1975) atribuye a los pensamientos (BECK 2012; CAMP 2004). Si una mente puede pensar que Juan ama a María, también puede pensar que María ama a Juan (Fodor I998, 139). Asimismo, si una mente puede pensar que María es feliz y Juan es austero, también puede pensar que María es austera y Juan es feliz. El requisito de generalidad hace referencia, en parte, al mismo fenómeno de la sistematicidad. Existe al menos una diferencia entre el requisito y el fenómeno de la sistematicidad. El primero es una propuesta conceptual acerca de cómo deben estar estructurados nuestros pensamientos. Evans (1982) entiende que es un principio ideal al cual los sistemas de pensamiento sólo se ajustan de manera aproximada. El segundo, en cambio, está planteado como un hecho empírico acerca de los pensamientos de las mentes humanas. Parece conceptualmente posible que haya mentes que aun pensando que Juan ama a María, no tengan la capacidad de pensar que María ama a Juan. Sin embargo, tener la habilidad de pensar el segundo pensamiento a partir del primero es "un rasgo de la vida mental de los humanos" (Fodor I 998, 49).

La explicación de la sistematicidad está dada, en parte, por una cuestión que atañe al vehículo de esos pensamientos, vehículo que Fodor entiende en términos del lenguaje del pensamiento. ${ }^{5}$ Así, los elementos mínimos del len-

${ }^{5}$ La sistematicidad se explica fundamentalmente por la composicionalidad sintáctica. Esto no implica que no intervenga la composicionalidad semántica. La sistematicidad es una propiedad del pensamiento que se explica en función de la composicionalidad semántica y sintáctica. Por un lado, un sistema de representaciones es semánticamente composicional en caso de que las propiedades semánticas de la representación compleja estén totalmente determinadas por las propiedades semánticas de sus constituyentes. Por otro lado, un sistema de representaciones es sintácticamente composicional en caso de que las propiedades sintácticas de la representación compleja estén totalmente determinadas por las propiedades sintácticas de sus constituyentes. Ver Fodor (2008) 
guaje del pensamiento (los conceptos) se recombinan según el requisito de generalidad. Evans, en cambio, cree que no hace falta comprometerse con la idea de que los pensamientos se vehiculan en lenguaje del pensamiento debido a que, como heredero de Wittgenstein, se aparta de la idea de que los conceptos son representaciones mentales. ${ }^{6}$ Desde la perspectiva fodoriana, en cambio, el requisito de generalidad hace alusión esencial a los vehículos representacionales del lenguaje del pensamiento. Creo que esta idea fodoriana es satisfactoria sólo parcialmente. En lo que sigue me ocuparé de los aspectos que considero correctos e incorrectos de la presentación fodoriana del requisito de generalidad.

El tratamiento que hace Fodor de la sistematicidad o requisito de generalidad parece mostrar la necesidad de vehículos linguaformes. Siguiendo a Fodor $(2007$; 2008), considero que las representaciones mentales cuyos vehículos son perceptivos no satisfacen el requisito. Creo que una buena razón para sostener esto es la siguiente. Si se admite que el requisito de generalidad se refiere a la forma de ciertos pensamientos, entonces las combinaciones a las que alude el requisito se hacen en virtud de la forma lógica de ciertos pensamientos. En términos muy generales, los pensamientos tienen una forma lógica subyacente que es una representación formal de su estructura lógica. La forma lógica expresa, de alguna manera, la estructura composicional del pensamiento (e.g., $F a, F b, G a, G b$ ) y lo hace explicitando la contribución que hace cada constituyente a la totalidad del pensamiento. Un defensor de los vehículos perceptivos, ¿puede dar cuenta de la forma lógica de los pensamientos?

La noción de "forma lógica" ha sido desarrollada desde un punto de vista filosófico y lingüístico (CARruthers 2003). Desde una perspectiva filosófica, la forma lógica de una oración es una construcción que contiene constantes lógicas, cuantificadores, variables y constantes de individuo. Desde una perspectiva lingüística, la forma lógica es una representación sintácticamente estructurada que es el resultado de los cómputos lingüísticos. La forma lógica junto con la forma fonética forman una descripción estructural que provee de instrucciones a los sistemas de actuación (СномSкY I 995). Ahora bien, independientemente de cómo entendamos la forma lógica, ésta no puede extraerse de las representaciones perceptivas.

La forma lógica puede extraerse de aquellas representaciones cuya descomposición es canónica. Por ejemplo, dadas las representaciones lingüísticas "María es dentista" y "Pedro es artista", ambas se pueden descomponer en términos singulares y predicados. Siempre que hacemos el ejercicio de descom-

\footnotetext{
${ }^{6}$ Davies (1998), es uno de los que ha argumentado que los neo-fregeanos, como Evans, están comprometidos con el lenguaje del pensamiento. Esto daría cuenta de la posibilidad de vincular las teorías de los conceptos concebidos como entidades abstractas con aquellas que asumen que son particulares mentales.
} 
poner estas representaciones encontramos los mismos componentes, términos singulares y predicados, y a estos componentes que siempre son los mismos en la descomposición de estas representaciones se los llama constituyentes canónicos. Como estas representaciones tienen constituyentes canónicos, se combinan de manera canónica en virtud de estos constituyentes y no en virtud de ningún otro tipo de constituyente. Las combinaciones canónicas que surgen de estas representaciones serían "María es artista" y "Pedro es dentista". Las representaciones de las cuales puede extraerse una forma lógica son las que tienen estas características, dado que la forma lógica hace explícita las relaciones entre los constituyentes canónicos de una representación (Fodor 2008).

Las imágenes mentales tienen características diferentes. Desarrollar este punto contribuirá a entender por qué las simulaciones no cumplirían con la condición de la sistematicidad. Pues, considérese que desde el enfoque enraizado la imaginería mental constituye uno de los mecanismos que opera haciendo uso de simulaciones (BARSALOU 2008). Una imagen mental (visual, motora o auditiva) es una representación consciente de un objeto ausente (BARSALOU I999A). Tomando como ejemplo a las imágenes visuales, las mismas no pueden descomponerse canónicamente. Se pueden descomponer de diversas maneras y en cada descomposición surgen componentes diferentes. Siguiendo un ejemplo de Fodor (2008), la foto de una persona (una imagen gráfica que tiene las mismas propiedades de las imágenes visuales) puede recortarse en partes de muchas maneras, y en cada recorte surgirían componentes diferentes de la foto. La descomposición de una imagen gráfica el carácter no canónico de sus partes. Dado que en las diferentes descomposiciones de una misma imagen pueden surgir diferentes componentes, estos componentes no serían constituyentes canónicos sino que, en cambio, serían meras partes a las que subyacen simulaciones. Por supuesto que las partes de una imagen visual pueden combinarse gracias al procesamiento subyacente de simulaciones. Siguiendo el ejemplo de la foto, los pedazos de la foto recortada se combinan para representar la imagen total de una persona. Pero como estas combinaciones se dan entre meras partes y no entre constituyentes canónicos, entonces las simulaciones subyacentes se combinarían de manera no canónica. De representaciones con estas características no se podría extraer ninguna forma lógica, lo cual significa que las imágenes visuales, a las cuales subyacen las simulaciones, no satisfacen el requisito de generalidad, el cual alude a las combinaciones realizadas en virtud de la forma lógica. Es cierto que Barsalou (I999A) considera que las simulaciones se combinan de manera sistemática pero tal como lo afirman Adams y Campbell (1999) el autor no explica de qué manera podrían satisfacer esta condición para la conceptualidad.

Habiendo desalentado la idea de que el requisito de generalidad se explica postulando simulaciones, creo que la posibilidad que queda es la que pone 
en evidencia Fodor (2007; 2008). Si los pensamientos como Juan es feliz tienen la forma lógica $F a$, esto es posible porque este pensamiento tiene una descripción canónica de la cadena de símbolos. La descripción canónica de Juan es feliz es $\left((J u a n)_{\mathrm{SN}}\left((\mathrm{es})_{\mathrm{V}}(\text { feliz) })_{\mathrm{SP}}\right)_{\mathrm{SV}}\right)_{\mathrm{O}}$. Esta descripción determina cuáles son los constituyentes canónicos del pensamiento Juan es feliz, eliminando constituyentes no canónicos como Juan es. Asimismo, la descripción canónica establece relaciones de jerarquía entre los constituyentes canónicos de Juan es feliz. Cada uno de estos constituyentes contribuye de diferente manera a la totalidad del pensamiento. El constituyente Juan, en tanto núcleo nominal, contribuye de manera diferente que el constituyente es, en tanto núcleo verbal.

La idea es que esta descripción canónica es posible si el vehículo de ese pensamiento es linguaforme. Sólo en un medio representacional linguaforme se pueden establecer constituyentes canónicos y relaciones jerárquicas entre los constituyentes de los pensamientos. El vehículo linguaforme permite una sintaxis combinatoria de los pensamientos, según la cual los constituyentes de los pensamientos tienen relaciones estructurales entre sí. Asimismo, los pensamientos vehiculados por representaciones linguaformes tienen una semántica composicional, según la cual sus constituyentes siempre hacen la misma contribución semántica en el pensamiento donde aparecen. La sintaxis combinatoria y la semántica composicional son características de los pensamientos que poseen constituyentes canónicos. Así, el requisito de generalidad requiere combinaciones que se hacen en base a la forma lógica de los pensamientos, la forma lógica de los pensamientos requiere una descripción canónica de sus componentes, la descripción canónica requiere que el vehículo representacional sea linguaforme, por lo tanto, el requisito de generalidad requiere que el vehículo representacional sea linguaforme.

Creo que es correcto considerar, junto con Fodor, que el requisito de generalidad nos compromete con un vehículo lingüístico. Sin embargo, considero incorrecto creer, como hace Fodor, que la conclusión anterior nos compromete de manera inmediata con la hipótesis del lenguaje del pensamiento. Un vehículo que tiene sintaxis combinatoria y semántica composicional es oracional. Así, también puede considerarse que los vehículos del lenguaje natural tienen algún tipo de sintaxis combinatoria y semántica composicional. ${ }^{7}$ De hecho,

${ }^{7}$ Fodor (200I), asumiendo que el pragmatismo radical respecto del lenguaje natural es correcto, sostiene que el lenguaje natural no es composicional. Según esta nueva perspectiva, solamente el lenguaje del pensamiento sería genuinamente composicional. Sin embargo, creo que este giro no es legítimo. Siguiendo a Clapp (2010), considero que si se admiten los argumentos pragmáticos radicales en contra de la composicionalidad del lenguaje natural, entonces también debería rechazarse la composicionalidad del lenguaje del pensamiento. Si el lenguaje natural no es composicional, Fodor dispondría de pocas herramientas para sostener que el lenguaje del pensamiento sí lo es. 
Fodor se inspira en las propiedades de los lenguajes naturales para caracterizar al lenguaje del pensamiento como teniendo sintaxis combinatoria y semántica composicional. En este sentido, creo que el requisito de generalidad exige que las representaciones tengan vehículos lingüísticos, y estos pueden ser o bien del lenguaje del pensamiento o bien del lenguaje natural.

Resumiendo, los conceptos son aquellas entidades que satisfacen de manera conjunta las dos condiciones que expuse a lo largo de este apartado. Cabe aclarar que la sistematicidad y la independencia del estímulo son propiedades conceptualmente independientes:

Una criatura [...] podría recombinar sistemáticamente sus representaciones aun cuando carezca de la libertad respecto del control del estímulo. A la inversa, podemos imaginar criaturas cuyos pensamientos fueran producidos por representaciones totalmente separadas e inestructuradas, en lugar de ser producidos por la interacción sistemática de sus constituyentes (representacionales), pero que pudiera tener todos sus pensamientos potenciales, cualquiera sea la circunstancia actual (CAMP 2009, 291)

Desde un punto de vista conceptual estas propiedades son independientes, pero "en la práctica están mucho más íntimamente relacionadas" (CAmp 2009, 291). Así, las representaciones conceptuales son las que se combinan sistemáticamente y son independientes del estímulo.

\section{Conclusión}

En un intento de elucidar la noción de "representación conceptual" como un aporte al debate enraizado, adopté un criterio funcional para la caracterización de la misma. Los roles funcionales básicos de los conceptos serían los siguientes: (i) se pueden combinar sistemáticamente para formar nuevas estructuras representacionales y (ii) se emplean en una variedad de tareas cognitivas con independencia del estímulo. Estas condiciones no son arbitrarias. Por el contrario, son condiciones que suelen aparecer en las propuestas psicológicas y filosóficas que caracterizan al contenido conceptual.

De modo que, las representaciones que son independientes del estímulo pero no pueden combinarse de manera sistemática no son conceptuales. Por ejemplo, tomemos el caso del estado propioceptivo en el cual un sujeto, después de una operación, aún siente dolor en su pierna amputada. El sujeto está en un estado en el cual siente que su miembro todavía está conectado al cuerpo y sigue funcionando con el resto de éste cuando en realidad es un 
miembro fantasma. Este estado representacional es independiente del estímulo porque se instancia sin que se requiera la presencia del estímulo proveniente del miembro en cuestión. En este caso la independencia del estímulo se da en tanto que la representación se activa aunque en el mundo no existe el estímulo pertinente. Pero sería difícil sostener que la representación que constituye este estado propioceptivo puede combinarse sistemáticamente con otras para dar origen a otros estados propioceptivos. Un estado propioceptivo posee la misma naturaleza que un estado perceptivo y, como hemos visto, los estados perceptivos no poseen constituyentes canónicos que puedan combinarse de manera sistemática.

Otro ejemplo es la conducta de los atáxicos ópticos. Los pacientes con ataxia óptica, aunque no tienen problemas perceptivos ni de orientación, no pueden alcanzar y tomar objetos presentados visualmente (RossetTi et al. 2003). Suele considerarse que la ataxia óptica es una patología de la coordinación visuomotora, la cual está involucrada en las acciones intencionales en las que el sujeto produce deliberadamente una secuencia de movimientos para alcanzar un objeto (Rossetti et al. 2003). Debido a este trastorno para apuntar hacia un target visual, se les vuelve difícil a los sujetos desenvolverse en tareas muy simples como prender un cigarrillo o tomar un vaso de agua. La ataxia óptica parece involucrar representaciones motoras independientes del estímulo. Aun cuando haya un estímulo distal presente, las representaciones motoras de los pacientes con ataxia óptica no están guiadas por ese estímulo. Pero estas representaciones no pueden combinarse sistemáticamente. La razón se debe a que los vehículos de las representaciones motoras no presentan la estructura de constituyentes canónicos que hemos visto.

Asimismo, las representaciones que pueden combinarse de manera sistemática y no pueden ser independientes del estímulo, tampoco serían conceptuales. Puede concebirse el requisito de generalidad de manera contrafáctica, es decir, según la restricción de la siguiente cláusula: "si ocurriera el estímulo relevante, entonces se combinarían las representaciones de la manera relevante" (CAMP 2009, 290). Esta cláusula restringe el dominio de combinaciones sistemáticas a las representaciones que un organismo activa en virtud del "estímulo relevante". Camp (2009) considera que ciertos animales con cognición básica satisfarían el requisito de generalidad bajo esta cláusula contrafáctica. Siguiendo un ejemplo presentado por Camp (2009, 280), el perro $D$ puede representarse al perro $M$ como amenazante y al perro $N$ como amistoso así como también puede representarse al perro $M$ como amistoso y al perro $N$ como amenazante. Estas combinaciones representacionales estarían restringidas por la información del medio ambiente, en el sentido de que se dan debido a la conducta efectiva que evidencian estos animales. De modo que, por ejemplo, 
si ocurriera que el perro $M$ evidenciara una conducta amenazante, entonces el perro $D$ lo representaría como amenazante. Lo importante a destacar es que este es un caso en el que se cumpliría el criterio de sistematicidad pero no se cumpliría el criterio de la independencia del estímulo para la conceptualidad. Así, según esta propuesta, las representaciones no conceptuales serían aquellas que no satisfacen en conjunto los criterios de independencia del estímulo y sistematicidad.

La idea que se asume en este artículo es que esta clarificación conceptual contribuiría a echar alguna luz sobre el debate metodológico enraizado en el cual se polemiza sobre la manera de integrar el enfoque enraizado con el clásico. El enfoque enraizado ofrece una explicación de las capacidades conceptuales en el cerebro apelando a las modalidades perceptivas, motoras, afectivas e introspectivas (BARSALOU 20I6). Esto no significa que haya necesariamente una reducción de nuestras capacidades conceptuales a los mecanismos sensorio-motores. El objetivo del enfoque enraizado sería entender cómo las modalidades así como también el ambiente físico y social contribuyen a la cognición pero dejando un espacio para las explicaciones que apelan a representaciones simbólicas (BARSALOU 2007). Buscar si hay univocidad o no en la noción de "representación conceptual" en primer lugar contribuiría a definir si efectivamente existe un acervo conceptual que compartan ambos enfoques. Esto es fundamental para vislumbrar si hay un verdadero debate o simplemente un diálogo de sordos entre partes. Como se desarrolló, las simulaciones sólo parcialmente responden a los criterios de conceptualidad elucidados dado que son independientes del estímulo pero no se combinan de manera sistemática. Aunque se necesitarían más elementos de juicio, podría pensarse que la manera de integrar estos enfoques es considerando que las representaciones simbólicas, que responden paradigmáticamente a las condiciones de conceptualidad analizadas, se complementan en la arquitectura cognitiva con las representaciones enraizadas no conceptuales. Otra posible interpretación es considerar que las simulaciones son entidades neurales en las que se implementan las funciones propias de las arquitecturas cognitivas clásicas. Johnson (2018) entiende que si bien Barsalou presenta su teoría en términos representacionales, bien podría entenderse como una propuesta neuropsicológica. Con todo, aún quedan muchos aspectos abiertos en este debate metodológico enraizado. 


\section{REFERENCIAS}

Adams y Campbell i 999, "Modaliy and Abstract Concepts", Behavioral and brain sciences 22: 577-660.

Barsalou, L. W. I999A, "Perceptual Symbol System", Behavioral and brain sciences 22: 577-660.

Barsalou, L. W. I999B, "Perceptions of Perceptual Symbols", Behavioral and brain sciences $22(4)$.

Barsalou, L. W. 2008, "Grounded Cognition”, Annu. Rev. Psychol. 59: 617-45.

Barsalou, L. W. 20io, "Grounded Cognition: Past, Present, and Future", Topics in Cognitive Science 2: 716-24.

Barsalou, L. W. 2016, "On Staying Grounded and Avoiding Quixotic Dead Ends", Psychon Bull Rev 23: 1122-42.

Barsalou, L. W. et al. 2007, "Cognition as Coordinated Non-cognition", Cognitive Processing 8: 79-91

Barsalou, L. W. I 999, "Perceptual Symbol Systems", Behavioral and Brain Sciences 22: 577-660.

Beck, J. 20I2, "The Generality Constraint and the Structure of Thought", Mind 121(483): 563-600.

Bermúdez, J. L. 2003, Thinking Without Words, Oxford, OUP.

Braine, M. D. S.; Reiser, B. J. y Rumain B. I984, "Some empirical justification for a theory of natural propositional reasoning", G. H. Bower (ed.) Psychology of Learning and Motivation, Orlando, Academic Press.

Camp, E. 2009, "Putting Thoughts to Work: Concepts, Systematicity, and Stimulus-Independence", Philosophy and Phenomenological Research 2: 275-311.

Camp, E. 2004, "The Generality Constraint: Nonsense and Categorial Restrictions", Philosophical Quarterly 54: 209-31.

Carruthers, P. 2003, “Thinking in Language? Evolution and a Modularist Possibility", P. Carruthers y J. Boucher (ed.), Language and Thought, Cambridge, CUP.

Chомsкy, N. 1995, El programa minimalista, Madrid, Alianza.

Clapp, L. 20 io, "Is even Thought Compositional?", Philosophical Studies 10.1007/ s11098-010-9649-2, Online First.

Conchiglia, G.; Della Rocca, G. y Grossi, D. 2007, "On a Peculiar Environmental Dependency Syndrome in a Case withFrontal-Temporal Damage: Zelig-like Syndrome", Neurocase 13: 1-5.

Davies, M. I998, "Language, thought and the language of thought (Aunty's own argument revisited)", P. CArruthers y J. Boucher (ed.) Language and Thought. Cambridge, CUP. 
Dove, G. O. 2008, "Beyond Perceptual Symbols: a Call for Representational Pluralism”, Cognition 110: 412-31.

Ellis, A. W. y Young, A. W. I992, Neuropsicología cognitiva humana, Barcelona, Masson.

Estes, W. K. I994, Classification and cognition, Oxford, OUP.

Evans, G. 1982, Varieties of Reference, Oxford, OUP.

FARA, M. 2006, "Dispositions", Standford Encyclopedia of Philosophy (edición primavera de 2006), E. N. Zalta (ed.), http://plato.stanford.edu/entries/dispositions/.

Fodor. J. 2008, LOT 2: The Language of Thought Revisited, Oxford, OUP.

Fodor, J. 2007, “The Revange of the Given”, B. P. McLaughlin y J. Cohen (ed.), Contemporary debates in philosophy of mind, Oxford, Blackwell.

Fodor, J. 200 I, "Language, Thought and Compositionality", Mind and Language 16: $1-15$.

Fodor, J. I998, Conceptos. Donde la ciencia cognitiva se equivocó, Barcelona, Gedisa. Fodor, J. 1975, El lenguaje del pensamiento, Madrid, Alianza.

Fodor, J. y Pylyshyn, Z. W. 1988, "Connectionism and Cognitive Architecture: a Critical Analysis”, C. McDonald y G. McDonald (ed.) (1995), Connectionism, MA, Blackwell.

Frege, G. I 892, “On Sense and Reference”, P. Geach y M. Black (ed.), Translations from the Philosophical Writings of Gottlob Frege, Oxford, OUP (I966).

Gentner, D. I983, "Structure-mapping: A theoretical framework for analogy", Cognitive Science 7: 155-70

Goldinger, S. D.; Papesh, M. H.; Barnhart, A. S.; Hansen, W. A. y Hout, M. C. 2016, "The poverty of embodied cognition", Psychon Bull Rev 23: 959-78.

Greene, J. D. 2009, "Dual-process morality and the personal/impersonal distinction: A reply to McGuire, Langdon, Coltheart, and Mackenzie", Journal of Experimental Social Psychology 45: 581-4.

Johnson, M. 20i 8, "The Embodiment of Language", Newen, A.; De Bruin, L. y Gallagher, S. (ed.), The Oxford Handbook of $4 E$ Cognition, Oxford, OUP.

Laurence, S. y Margolis, S. (ed.) i 999, Concepts. Core Readings, Cambridge, MA, MIT Press.

Lhermitte, M. D. I986, "Human autonomy and the frontal lobes. Part II: Patient behavior in complex and social situations: The "environmental dependency syndrome", Annals of Neurology 19: 335-43.

Louwersen, M. 20 I 2, "Symbol Indeterminacy in Symbolic and Embodied Cognition", Topics in Cognitive Science 3: 273-302.

Machery, E. 2009, Doing Without Concepts, Nueva York, OUP.

Mahon, B. Z. 2015 , "What is embodied about cognition?", Language, Cognition and Neuroscience 30: 420-9. 
MARTIN, A. 20I6, "GRAPES—Grounding representations in action, perception, and emotion systems: How object properties and categories are represented in the human brain", Psychon Bull Rev 23: 979-90.

Millikan, R. 2000, On Clear and Confused Ideas: An Essay about Substance Concepts, Cambridge, CUP.

Monti, M.; Lawrence, M. y Osherson, D. 2009, "The Boundaries of Language and Thought in Deductive Inferences", PNAS 106, 125554-9.

Pecher, D. 20I3, "The Perceptual Representation of Mental Categories", D. Reisberg (ed.), The Oxford Handbook of Cognitive Psychology, Oxford, OUP.

Piccinini, G. y Sсотт, S. 2006, "Splitting Concepts", Philosophy of Science 73: 390-409.

Prinz, J. 2004, Gut Reactions. A Perceptual Theory of Emotions, Oxford, OUP.

Prinz, J. 2002, Furnishing the Mind. Concepts and their Perceptual Basis, Cambridge, MA, MIT Press.

Rehder, B. 2003, "A causal-model theory of conceptual representation and categorization", Journal of Experimental Psychology Learning, Memory, and Cognition 29: 1141-59.

Rosh, E. I999, "Principles of Categorization”, Laurence, S. y Margolis, E. (ed.), Concepts. Core Readings, Cambridge, MA, MIT Press.

Rosh, E.; Mervis, C. B.; Gray, W. D.; Johnson, D. M. y Boyes-Braem, P. I978, "Basic Objects in Natural Categories", Cognitive Psychology 8: 382-439.

Rossetti, Y.; Pisella. L. y Vighett, A. 2003, "Optic Ataxia Revisited”, Experimental Brain Research 153: 171-9.

RyLe, G. I949, El concepto de lo mental, Buenos Aires, Paidós.

Smith, E. y Medin, D. L. I98 I, Categories and Concepts, Cambridge, Harvard University Press.

Weber, A. y Vosgerau, G. 20 i 8, "Critical Note", Newen, A., De Bruin, L. y Gallagher, S. (ed.) The Oxford Handbook of $4 E$ Cognition, Oxford, OUP.

Weiskopf, D. (ms.) "Concepts as a Functional Kind".

ZwaAn, R. 2014, "Embodiment and Language Comprehension: Reframing the Discussion", Trends in Cognitive Sciences 18: 229-34. 
